•生物多样性监测网络专题・

\title{
鱼类多样性监测的理论方法及中国内陆 水体鱼类多样性监测
}

\author{
刘焕章 ${ }^{1}$ 杨君兴 $^{2}$ 刘淑伟 ${ }^{2}$ 高 欣 ${ }^{1}$ 陈宇顺 ${ }^{1}$ 张春光 ${ }^{3}$ \\ 赵 凯 ${ }^{4}$ 李新辉 ${ }^{5}$ 刘 伟 6 \\ 1 (中国科学院水生生物研究所, 武汉 430072) \\ 2 (中国科学院昆明动物研究所, 昆明 650223) \\ 3 (中国科学院动物研究所, 北京 100101) \\ 4 (中国科学院西北高原生物研究所, 西宁 810008) \\ 5 (中国水产科学研究院珠江水产研究所, 广州 510380) \\ 6 (中国水产科学研究院黑龙江水产研究所, 哈尔滨 150076)
}

\begin{abstract}
摘要: 近年来, 生物多样性监测网络的建设得到广泛重视, 全球、地区或国家生物多样性观测网不断组建。生物 多样性观测的理论框架得到发展, 提出了生物多样性核心监测指标(Essential Biodiversity Variables, EBV)。鱼类多 样性监测的理论框架包含于生物多样性核心监测指标之内，在遗传、物种、生态系统等多层次进行。基于鱼类监 测提出的生物完整性指数(index of biotic integrity, IBI)强调不同物种的生态功能, 可以综合反映群落结构和功能的 变化, 得到广泛应用。鱼类多样性的监测方法是传统网具和现代水声学等方法的结合。监测结果的分析可以进行 简单的指数比较, 也可以进行长期的趋势分析, 寻找关键节点, 探讨宏观生态格局的变化。中国内陆水体鱼类多样 性监测网隶属于中国生物多样性监测与研究网络, 拟选取长江、黄河、黑龙江、珠江、澜沧江、怒江、塔里木河 及青海湖 8 大流域, 对 25 个重要区域和 24 个重点物种(类群)进行监测, 从重要区域鱼类群落结构、重点物种(类群) 种群动态和个体生物学特征、遗传多样性、早期资源等不同层次, 全面监测我国内陆水体鱼类生物多样性状况。 关键词: 内陆水体鱼类; 监测网络; 物种多样性; 生物学特征
\end{abstract}

\section{Theory and methods on fish diversity monitoring with an introduction to the inland water fish diversity observation in China}

Huanzhang $\mathrm{Liu}^{1 *}$, Junxing Yang${ }^{2}$, Shuwei $\mathrm{Liu}^{2}$, Xin Gao ${ }^{1}$, Yushun Chen ${ }^{1}$, Chunguang Zhang ${ }^{3}$, Kai Zhao ${ }^{4}$ Xinhui Li ${ }^{5}$, Wei Liu ${ }^{6}$

1 Institute of Hydrobiology, Chinese Academy of Sciences, Wuhan 430072

2 Kunming Institute of Zoology, Chinese Academy of Sciences, Kunming 650223

3 Institute of Zoology, Chinese Academy of Sciences, Beijing 100101

4 Northwest Institute of Plateau Biology, Chinese Academy of Sciences, Xining 810008

5 Pearl River Fisheries Research Institute, Chinese Academy of Fisheries Sciences, Guangzhou 510380

6 Heilongjiang Fisheries Research Institute, Chinese Academy of Fisheries Sciences, Harbin 150076

\begin{abstract}
In recent years, the establishment of biodiversity observation networks (BON) has been of great concern. The global scale GEO-BON (Global Earth Observation—Biodiversity Observation Network), regional EBONE (European Biodiversity Observation Network) and AP BON (Asia-Pacific BON), and local networks such as the J-BON (Japanese BON) and French BON have been successful. The introduction of Essential Biodiversity Variables (EBV) has laid a theoretical foundation for biodiversity observations. The fish biodiversity observation theory is embedded in the EBV, and includes work at the genetic, species, and ecosystem levels. Originally designed for fish monitoring, the index of biotic integrity (IBI) has become the
\end{abstract}


most popular index, and emphasizes the identification of different ecological functional groups, which can reflect changes in community structure and function. Fish diversity survey methods include both traditional nets and modern instruments such as a hydroacoustic sonar system. Analysis of monitoring data can be completed as simple comparisons of various indices, modeling long term trends to identify change-points, and exploring ecological regime shifts. As a part of the Chinese Biodiversity Monitoring and Research Network (Sino BON) - Inland Water Fish is designed to conduct fish monitoring work in 8 major drainage basins in China including the Yangtze River, the Yellow River, the Heilongjiang River, the Zhujiang River, the Lancang River, the Nujiang (Salween) River, the Tarim River, and the Qinhaihu Lake. A total of 25 focused areas and 24 targeted species (groups) have been selected as sampling sites and crucial indicators, respectively, and monitoring variables including community structure, population structure and dynamics, biological traits, genetic diversity, and fish early resources.

Key words: inland water fish; Sino BON; species diversity; biological traits

近年来，人类活动的影响造成了生物多样性的 丧失, 特别是水生生态系统中鱼类物种的濒危与灭 绝, 物种多样性下降, 鱼类小型化, 生态系统结构、 功能发生变化, 遗传多样性减少等(陈宜瑜, 1990; Sarkar et al, 2012)。为了保护生物多样性, 遏制其下 降的趋势, 许多国际组织和各国政府采取了诸多措 施, 特别是建立观测网络, 监测生物多样性的多项 指标, 评估生物多样性的状况。当前, 广泛关注的 一项工作是在地球观测组织(Global Earth Observation, GEO)框架内，联合IUCN、BIODIVERSITAS等 多个国际组织形成的生物多样性观测网络 (Biodiveristy Observation Network, BON) (Scholes et al, 2008, 2012)。该组织致力于建立一个全球性的科 学框架, 以观测生物多样性的变化, 包括收集生物 多样性的资料, 从事长时间的连续观测, 进行预测 和相关分析。在GEO BON的框架建议被提出以后, 得到了世界各国政府和非政府组织的响应, 并提出 了多个地区性的观测网络, 如欧洲观测网络 (EBONE)、亚太观测网络 (Asia-Pacific BON, AP $\mathrm{BON})$, 以及多个国家的观测网络, 例如日本的 J-BON, 法国的French BON等(Scholes et al, 2012; Nakano et al, 2014)。这些观测网络期望通过整体的 合作, 切实地了解和保护生物多样性。特别值得高 兴的是，2014年在中科院创新项目的支持下，成立 了中国生物多样性监测与研究网络(Biodiversity Observation Network of China, Sino BON) (马克平, 2015), 包含 10 个专项网和 1 个综合监测管理中心, 以期对中国的生物多样性进行全面的监测和研究。

鱼类是生物多样性的一个重要组分。据FishBase的统计, 全世界的鱼类物种数已达 3 万多种
(Froese \& Pauly, 2016), 它们在全球生态系统中起 着极其重要的作用。新成立的中国生物多样性监测 与研究网络中也包含对鱼类的监测, 即内陆水体鱼 类多样性监测网(Sino Bon-Inland Water Fish)。本文 拟综述世界鱼类多样性监测的理论基础和方法, 介 绍中国内陆水体鱼类多样性监测网的设计框架和 拟进行的工作, 期望藉此推进我国鱼类多样性监测 和保护工作的开展。

\section{1 鱼类多样性监测的理论与方法}

\section{1 鱼类多样性监测的指标体系}

在GEO BON的框架建议被提出以后, 为配合 相关的工作, Pereira等(2013)提出了配套的生物多 样性核心变量 (Essential Biodiversity Variables, EBV), 期望从遗传、物种、群落、生态系统结构、 功能等多尺度反映生物多样性的变化(表1)。在这一 体系中, 既包括我们常规使用的遗传多样性、物种 数目、群落物种多样性等内容, 也包含了物种的特 征, 例如植物叶子的颜色变化时间, 类比于鱼类的 生长、繁殖状况等。我们认为这一指标体系将会对 GEO BON的监测工作起到重要的理论指导作用。

在很多情况下, 为了反映某地区生物多样性的 总体状况, 生物多样性的监测非常强调群落层次物 种多样性的监测。传统的群落物种多样性衡量方法 是计算各种生物多样性指数, 如Shannon-Wiener指 数、Simpson多样性指数等。但是, 有学者认为, 单 纯计算笼统的生物多样性指数仅能反映物种的总 体状况, 而忽略了群落中物种的生态类型和生态功 能的变化(Karr, 1981)。因此, Karr (1981)提出了生物 完整性指数(index of biotic integrity, IBI)的指标体系 
表1 生物多样性的核心变量说明(引自Pereira et al, 2013)

Table 1 Examples of candidate Essential Biodiversity Variables (EBV) (adopted from Pereira et al, 2013)

\begin{tabular}{|c|c|c|c|}
\hline$\overline{\mathrm{EBV}}$ 类别 & EBV举例 & 度量与尺度 & 时间敏感性 \\
\hline EBV class & EBV examples & Measurement and scalability & Temporal sensitivity \\
\hline 遗传组成 & 基因型多样性 & 选定的物种(濒危或家养物种)在代表性分布区的基因型 & 世代时间 \\
\hline Genetic composition & Allelic diversity & $\begin{array}{l}\text { Genotypes of selected species (e.g. endangered, domesticated) at } \\
\text { representative locations }\end{array}$ & Generation time \\
\hline 物种种群 & 丰度或分布 & 进行计数或出现与否调查, 主要针对大范围网络尺度上容易监测的 & 1年-10年以上 \\
\hline Species populations & $\begin{array}{l}\text { Abundances and } \\
\text { distributions }\end{array}$ & $\begin{array}{l}\text { 物种、生态系统服务重要的物种等 } \\
\text { Counts or presence surveys for groups of species easy to monitor or } \\
\text { important for ecosystem services (ES), over a extensive network of sites }\end{array}$ & 1 to $>10$ years \\
\hline $\begin{array}{l}\text { 物种特征 } \\
\text { Species traits }\end{array}$ & 形态学 Phenology & $\begin{array}{l}\text { 遥感监测植物叶子颜色变化的时间, 需要现场核实。在鱼类可以采用 } \\
\text { 生长、繁殖等特征 } \\
\text { Timing of leaf coloration by remote sensing (RS), with in situ validation. } \\
\text { Growth and reproduction traits in fish. }\end{array}$ & $\begin{array}{l}1 \text { 年 } \\
1 \text { year }\end{array}$ \\
\hline $\begin{array}{l}\text { 群落组成 } \\
\text { Community composition }\end{array}$ & $\begin{array}{l}\text { 分类单元多样性 } \\
\text { Taxonomic diversity }\end{array}$ & $\begin{array}{l}\text { 多个分类单元的调查以及选定区域的宏基因组研究 } \\
\text { Consistent multitaxa surveys and metagenomics at select locations }\end{array}$ & $\begin{array}{l}5 \text { 年 }-10 \text { 年以上 } \\
5 \text { to }>10 \text { vears }\end{array}$ \\
\hline 生态系统结构 & 生境结构 & 全球或区域尺度的生物量或覆盖度遥感 & $1-5$ 年 \\
\hline Ecosystem structure & Habitat structure & RS of cover (or biomass) by height (or depth) globally or regionally & 1 to 5 years \\
\hline 生态系统功能 & 营养物质保留 & 选定区域的营养物输出/输入比例测量 & 1年 \\
\hline Ecosystem function & Nutrient retention & Nutrient output/input ratios measured at select locations & 1 year \\
\hline
\end{tabular}

表2 基于生物完整性指数(IBI)评价鱼类群落生物完整性的指标及评分级别(修改自Karr, 1981, 1991)

Table 2 Metrics used to assess biological integrity of fish communities based on the Index of Biotic Integrity (IBI) (from Karr, 1981, 1991 with modifications)

\begin{tabular}{|c|c|c|c|}
\hline \multirow{2}{*}{$\begin{array}{l}\text { 评价指标 } \\
\text { Metrics }\end{array}$} & \multicolumn{3}{|c|}{ 评分级别 Rating of metrics } \\
\hline & 5 & 3 & 1 \\
\hline \multicolumn{4}{|l|}{ A. 物种组成与丰富度 Species richness and composition } \\
\hline 1. 鱼类物种总数(土著物种) Total number of fish species (native fish species) & \multirow{5}{*}{\multicolumn{3}{|c|}{$\begin{array}{l}\text { 大型河流鱼类物种期望值高; 中 } \\
\text { 国的河流鲤科鱼类物种多。 } \\
\text { Expectations for metrics 1-5 vary } \\
\text { with stream size and region. Large } \\
\text { rivers are with more species, and } \\
\text { more cyprinids in Chinese waters }\end{array}$}} \\
\hline 2. 鲇类物种单元与数量(底栖物种) Number and identity of darter species (benthic species) & & & \\
\hline 3. 太阳鱼科物种单元与数量(中层鱼类) Number and identity of sunfish species (water-column species) & & & \\
\hline 4. 亚口鱼科物种单元与数量(长寿命鱼类) Number and identity of sucker species (long-lived species) & & & \\
\hline 5. 非耐受型鱼类物种单元与数量 Number and identity of intolerant species & & & \\
\hline $\begin{array}{l}\text { 6. 蓝绿鳞鳃太阳鱼个体组成百分比(耐受型鱼类) Percentage of individuals as green sunfish (tolerant } \\
\text { species) }\end{array}$ & $<5$ & $5-20$ & $>20$ \\
\hline \multicolumn{4}{|l|}{ B. 营养类型组成 Trophic composition } \\
\hline 7. 杂食性鱼类个体组成百分比 Percentage of individuals as omnivores & $<20$ & $20-45$ & $>45$ \\
\hline 8. 昆虫食性鲤科鱼类个体组成百分比 Percentage of individuals as insectivorous cyprinids (insectivores) & $>45$ & $45-20$ & $<20$ \\
\hline 9. 凶猛肉食性鱼类个体组成百分比(顶级捕食者) Percentage of individuals as piscivores (top carnivores) & $>5$ & $5-1$ & $<1$ \\
\hline \multicolumn{4}{|l|}{ C. 鱼类丰度与状况 Fish abundance and condition } \\
\hline 10. 采集到的样本个体数 Number of individuals in sample & \multicolumn{3}{|c|}{$\begin{array}{l}\text { 指标 } 10 \text { 随河流大小等因子变化 } \\
\text { Expectations for metric } 10 \text { vary } \\
\text { with stream size and other factors }\end{array}$} \\
\hline 11. 杂交个体百分比 Percentage of individuals as hybrids & 0 & $>0-1$ & $>1$ \\
\hline $\begin{array}{l}\text { 12. 带病、肿瘤、鯺条损伤、或骨骼畸形的个体百分比 } \\
\text { Percentage of individuals with disease, tumors, fin damage, and skeletal anomalies }\end{array}$ & $0-2$ & $>2-5$ & $>5$ \\
\hline
\end{tabular}

(表2)。该指标体系强调不同物种的生态功能，其内 容包括总的物种数目、不同生态类型鱼类物种数, 以及受影响的物种的数量等。因此IBI指数可以综合 反映群落结构和功能的变化。该指标体系提出以后, 立刻得到积极响应, 并得到各种修正和改进, 不仅 适用于鱼类的监测分析, 也被用于底栖动物等类群 的分析。目前, 许多地区和单位在进行河流的监测
时, 均以IBI指数作为基本的指标, 使得该指标体系 成为当前使用最广泛的指标体系。

实际监测工作中, 不同的人类活动产生的影响 是不一样的, 监测的指标内容也需要进行适当的调 整。如大坝的修建、鱼类栖息地的改变等会导致鱼 类群落结构的变化, IBI指数应该是合适的选择; 捕 捞压力过大会造成鱼类的小型化, 因此监测的内容 
表3 鱼类调查方法及其适用水体(修改自Giles et al, 2005)

Table 3 Methods for fish surveying and applied water body (From Giles et al, 2005 with modifications)

\begin{tabular}{ll}
\hline 调查方法 Survey methods & 适用水环境 Applied water body \\
\hline 目测调查 Visual surveys & 小型水体或清澈的溪流 Small pools and clear streams \\
渔获物调查 Catch returns & 流水或静水水体 Running and still waters \\
定置网等诱捕型网具 Traps & 流水或静水水体 Running and still waters \\
撒网等网具 Lift, throw and push netting & 流水或静水水体 Running and still waters \\
电鱼 Electrofishing & 流水或静水水体 Running and still waters \\
刺网 Gill netting & 缓流或静水水体 Slow-flowing or still waters \\
围网 Seine netting & 缓流或静水水体 Slow-flowing or still waters \\
拖网 Trawl netting & 缓流或静水水体 Slow-flowing or still waters \\
水声学计数 Hydroacoustic sonar counters & 缓流或静水水体 Slow-flowing or still waters \\
电子计数 Electronic counters & 流水水体 Running waters \\
\hline
\end{tabular}

应该增加物种特征，如个体的生长情况等; 近亲繁 殖可能造成遗传多样性的丧失, 不同分子标记的遗 传多样性监测与分析就是必不可少的内容。

\section{2 鱼类多样性监测的采样方法}

目前鱼类监测系统中比较系统的工作有美国 陆军工程兵团 (US Army Corps of Engineers, USACE)、美国地质调查局(US Geological Survey, USGS)、美国鱼类与野生动物局(US Fish and Wildlife Service, USFWS)及相关单位开展的密西西 比河干流及主要支流的监测工作(Barko et al, 2004; Killgore et al, 2007; Steuck et al, 2010; Miranda \& Killgore, 2013)。另外在加拿大、欧洲、澳大利亚等 国家和地区, 也有很多的鱼类监测系统。在进行这 些监测工作时, 许多部门提出了非常具体的监测方 法或手册, 特别是《生物多样性工作手册》 (Handbook of Biodiversity Methods)中对鱼类监测方 法有详细和具体的描述(Giles et al, 2005)(表3)。这些 方法有传统的渔具, 如刺网、拖网、各种诱捕网具 (定置网、地笼、虾笼) 等, 也有现代的鱼探仪等水声 学设备, 在不同的水环境(河流、溪流、湖泊)条件下, 以及服务于不同目的(物种识别、种群估算)的时候, 可以分别参考使用。此外, 最近中国环保部也颁布 了生物多样性观测技术导则 - 内陆水域鱼类 $(\mathrm{HJ}$ 710.7-2014), 也可以供参考使用。

\section{3 多样性观测数据的分析方法}

在获得到了大量的监测数据以后, 如何分析、 评价这些数据, 并对未来的工作进行指导, 是生物 监测需要解决的重要问题。最简单的办法是对获得 的评价结果进行直接的对比。例如, Miller等(1988) 对美国加州Fresno河Hidden大坝下游某地点1970-
1985年的调查数据进行IBI评分，比较结果发现该 地的评分级别由1970年的良好变成1985年的一般。 从评分内容也可以看出, 土著鱼类百分比变化尤其 大，反映出土著鱼类受到了极大的影响。

随着生物多样性监测工作的开展, 许多学者对 生物多样性长期变化趋势和变化节点进行了研究。 例如, Fewster等(2000)利用the British Trust for Ornithology's Common Birds Census (CBC) 的调查数 据, 对12种鸟类的丰度变化趋势和关键变化点进行 了分析, 发现这些物种的丰度存在增加和减少的关 键变化点(图1)。

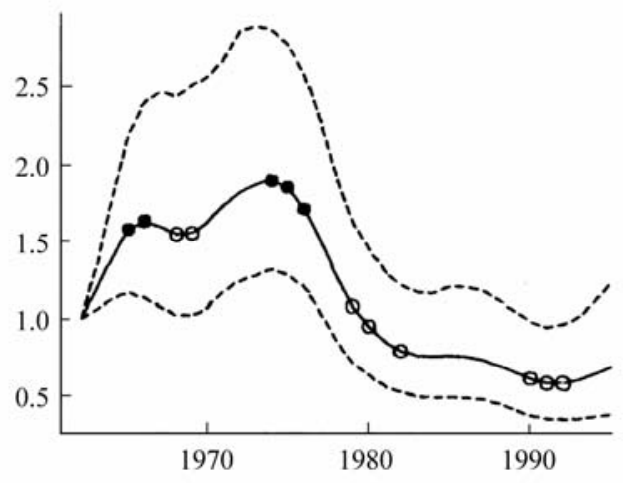

图1 基于不列颠鸟类调查数据库分析的红腹灰雀丰度指数 变化趋势图示(实线为丰度指数变化曲线, 虚线为 $95 \%$ 置信 区间, 黑点表示上升的变化点, 圆圈表示下降的变化点, 引 自Fewster et al, 2000)

Fig. 1 Index curves from CBC data for the Bullfinch. The solid lines give the index curves from a GAM and the dashed lines represent upper and lower 95\% bootstrapped confidence limits. A solid circle denotes a point at which the second derivative is significantly negative (a downturn in the index curve), and an open circle denotes a point at which the second derivative is significantly positive (an upturn in the index curve) (Fewster et al,, 2000). 
由于生物多样性不是孤立存在, 而是存在于生 态系统之中的, 生态系统的稳态转换也可以用来分 析生物多样性的变化情况。例如, Hare和Mantua (2000)对北太平洋(North Pacific Ocean)的100个环 境因子(包括31个气候因子和69个生物因子)分析, 发现其在1977和1989年有两次明显的稳态转换, 特 别是在1989年的稳态转换中, 生物的转换非常明显 (图2)。

生物多样性监测的目的是发现和评价监测指 标的变化。如果相关指标发生了稳态转换, 则意味 着生物多样性的大格局发生了变化。如果是群落特 征发生变化, 说明群落结构功能进入了新的格局; 如果是物种特征发生变化, 说明物种的生活史过程
进入了新的格局。在这种情况下, 相关的研究和管 理需要采取不同的措施。

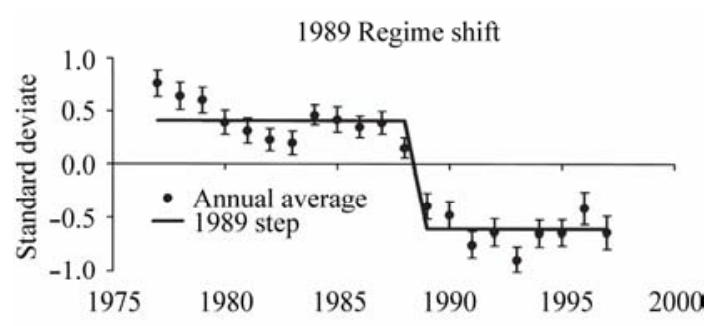

\section{图2 基于100个环境因子分析的北太平洋生态稳态转换(引} 自Hare \& Mantua, 2000)

Fig. 2 Regime shift analyses of a composite of the 100 environmental time series in North Pacific Ocean (Hare \& Mantua, 2000)

表4 中国内陆水体鱼类多样性监测专项网空间布局、重要地区、重点物种及主要承担单位

Table 4 The scheme of the Sino BON-Inland Water Fish, including focused areas, targeted species (groups) and lead institutions

\begin{tabular}{|c|c|c|c|}
\hline 水系 & 重要地区 & 重点物种 & 主要承担单位 \\
\hline Water systems & Focused areas & Target species & Lead institutions \\
\hline 长江 & 长江上游珍稀特有鱼类保护区(四川合江)、三峡 & 洄游性鱼类(鲟鱼类)、长江上游特有鱼 & 中国科学院水生生物研究所 \\
\hline Yangtz River & $\begin{array}{l}\text { 库尾(重庆)、三峡大坝坝下(湖北宜昌)、中游湖 } \\
\text { 泊区(江西湖口) } \\
\text { Reserve for rare and endemic fishes of the Upper } \\
\text { Yangtz River (Hejiang), end of the Three Gorges } \\
\text { Reservoir (Chongqing), downstream of the Three } \\
\text { Gorges Dam (Yichang), and floodplain of the } \\
\text { middle Yangtz (Hukou) }\end{array}$ & $\begin{array}{l}\text { 类(圆口铜鱼)、长江重要经济鱼类(四 } \\
\text { 大家鱼) } \\
\text { Anadromous fish (sturgeons), endemic } \\
\text { fishes of the Upper Yangtz River, eco- } \\
\text { nomic species (the four major Chinese } \\
\text { carps) }\end{array}$ & $\begin{array}{l}\text { Institute of Hydrobiology, Chinese } \\
\text { Academy of Sciences }\end{array}$ \\
\hline 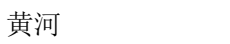 & 上游(巴彦淖尔)、中游(三门峡)、下游(东营) & 马口鱼或宽鯺鱲、裂腹鱼类、鲇类 & 中科院动物研究所 \\
\hline Yellow River & $\begin{array}{l}\text { Upper reaches (Bayan Nur), middle reaches } \\
\text { (Sanmenxia), and lower reaches (Dongying) }\end{array}$ & $\begin{array}{l}\text { Opsariicjthys bidens or Zacco platypus, } \\
\text { schizothroaxins, and catfishes }\end{array}$ & $\begin{array}{l}\text { Institute of Zoology, Chinese } \\
\text { Academy of Sciences }\end{array}$ \\
\hline 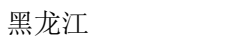 & 上游(呼玛)、中游(夢北)、下游(抚远) & 施氏鲟或达氏鳇、鲑科鱼类 & 中国水产科学研究院黑龙江水产 \\
\hline Heilongjiang River & $\begin{array}{l}\text { Upper reaches (Huma), middle reaches (Luobei), } \\
\text { and lower reaches (Fuyuan) }\end{array}$ & $\begin{array}{l}\text { Acipenser schrenckii, Huso dauricus, } \\
\text { salmonids }\end{array}$ & $\begin{array}{l}\text { 研究所 } \\
\text { Heilongjiang River Fisheries Re- } \\
\text { search Institute of Chinese Acad- } \\
\text { emy of Fishery Sciences }\end{array}$ \\
\hline 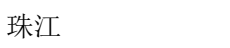 & 上游(合山)、中游(桂平)、下游(肇庆) & 广东鲂、野鲮亚科代表种、鳅类代表 & 中国水产科学研究院珠江水产研 \\
\hline Zhujiang River & $\begin{array}{l}\text { Upper reaches (Heshan), middle reaches (Guip- } \\
\text { ing), and lower reaches (Zhaoqing) }\end{array}$ & $\begin{array}{l}\text { 种 } \\
\text { Megalobrama terminalis, labeonins, } \\
\text { loaches }\end{array}$ & $\begin{array}{l}\text { 究所 } \\
\text { Zhujiang River Fisheries Research } \\
\text { Institute of Chinese Academy of } \\
\text { Fishery Sciences }\end{array}$ \\
\hline 澜沧江 & 上游(维西)、中游(大理)、下游(景洪) & 裂腹鱼类、鮡类、鲃类代表种 & 中科院昆明动物研究所 \\
\hline Lancang River & $\begin{array}{l}\text { Upper reaches (Weixi), middle reaches (Dali), and } \\
\text { lower reaches (Jinghong) }\end{array}$ & Schizothroaxins, sisorins, barbinins & $\begin{array}{l}\text { Kunming Institute of Zoology, } \\
\text { Chinese Academy of Sciences }\end{array}$ \\
\hline 怒江 & 上游(贡山)、中游(六库)、下游(永德) & 裂腹鱼类、鮡类、鳅类的代表种 & 中科院昆明动物研究所 \\
\hline Nujiang River & $\begin{array}{l}\text { Upper reaches (Gongshan), middle reaches } \\
\text { (Liuku), lower reaches (Yongde) }\end{array}$ & Schizothroaxins, sisorins, loaches & $\begin{array}{l}\text { Kunming Institute of Zoology, } \\
\text { Chinese Academy of Sciences }\end{array}$ \\
\hline 塔里木河 & 上游(阿拉尔)、中游(沙雅)、下游(尉犁) & 裂腹鱼类、鳅科鱼类代表种 & 中科院西北高原生物研究所 \\
\hline Tarim River & $\begin{array}{l}\text { Upper reaches (Alaer), middle reaches (Shaya), } \\
\text { and lower reaches (Yuli) }\end{array}$ & Schizothroaxins, loaches & $\begin{array}{l}\text { Northwest Institute of Plateau } \\
\text { Biology, Chinese Academy of } \\
\text { Sciences }\end{array}$ \\
\hline 青海湖 & 湖西北岸(刚察)、湖北岸(海晏)、湖西南岸(共和) & 裂腹鱼类、鳅科鱼类代表种 & 中科院西北高原生物研究所 \\
\hline Qinghai Lake & $\begin{array}{l}\text { Northwest bank (Gangcha), north bank (Haiyan), } \\
\text { and south bank (Gonghe) }\end{array}$ & Schizothroaxins, loaches & $\begin{array}{l}\text { Northwest Institute of Plateau } \\
\text { Biology, Chinese Academy of } \\
\text { Sciences }\end{array}$ \\
\hline
\end{tabular}




\section{2 中国内陆水体鱼类多样性监测网介绍}

\section{1 总体设计}

中国内陆水体鱼类多样性监测专项网将在长江 等 8 大水系选择 25 个重要地区, 对鱼类多样性的总体 变化情况进行监测, 并选择24个区域代表性物种(类 群), 监测它们的主要生物学特征状况。具体的重要地 区、重点物种以及主要承担单位的信息见表4、图3。

8大河流选择的依据是：它们代表了我国不同 的河流类型、不同的水环境条件, 它们纵横交错, 拥有丰富的鱼类多样性及渔业资源, 形成了我国流 域范围内的核心网络。除长江流域有 4 个重要地区 外, 其他流域均为3个, 合计 25 个重要地区。每一水 系3个重点物种(类群), 合计 24 个重点物种(类群)。 重点类群的选择依据是: 它们为其分布水系的代表 性类群, 同时考虑优势类群和生态功能代表性。如 长江流域选择了洄游性鱼类(鲟鱼类)、长江上游特 有鱼类(圆口铜鱼 Coreius guichenoti)、长江重要经 济鱼类(四大家鱼)。监测的内容包括群落、物种、 遗传等不同层次的内容(表5)。监测内容在生物多样 性核心变量(EBV)的框架下, 结合IBI的理论, 以及 鱼类生物学特征进行了具体化, 在群落层次上强调 了物种数目、不同的生态功能类群; 在物种层次上,
强调了个体生长、繁殖等特征。对这些特征的分析 应该可以全面反映鱼类多样性的现状、发生的变化, 并寻找导致变化的原因。

\section{2 科学目标}

中国内陆水体鱼类多样性监测专项网将通过 多家单位的合作, 进行长期的监测, 实现其科学目 标。首先, 获取重要区域鱼类资源状况的第一手资 料。在长江、黄河、黑龙江、珠江、澜沧江、怒江、 塔里木河及青海湖8大流域选取代表性区域，建立 鱼类监测和研究平台, 运用渔获物调查、水环境监 测等方法, 结合水下机器人视频追踪、鱼探仪探测、 声学信标监测等技术，获取各流域鱼类生物多样性 总体概况的基本数据, 包括总体的物种组成、总体 的资源量状况、优势种的组成、不同分类单元和功 能类群的组成、外来种的组成等, 以及相关的环境 因子参数。

其次, 分析重点监测对象的生物学特征。代表 性物种的状况可以反映生态系统的健康状况和环境 的影响, 是生物多样性监测的重要内容。内陆水体鱼 类专项网将重点监测各流域代表性物种, 获取大量 第一手资料, 分析它们的种群数量、年龄结构、个体 大小、繁殖时间、繁殖群体组成、早期资源量(即 繁殖的后代数量)等特征，并将采用线粒体DNA、

\section{表5 中国内陆水体鱼类多样性监测专项网的监测内容、方法和主要指标}

Table 5 Monitoring variables and methods of the Sino BON-Inland Water Fish

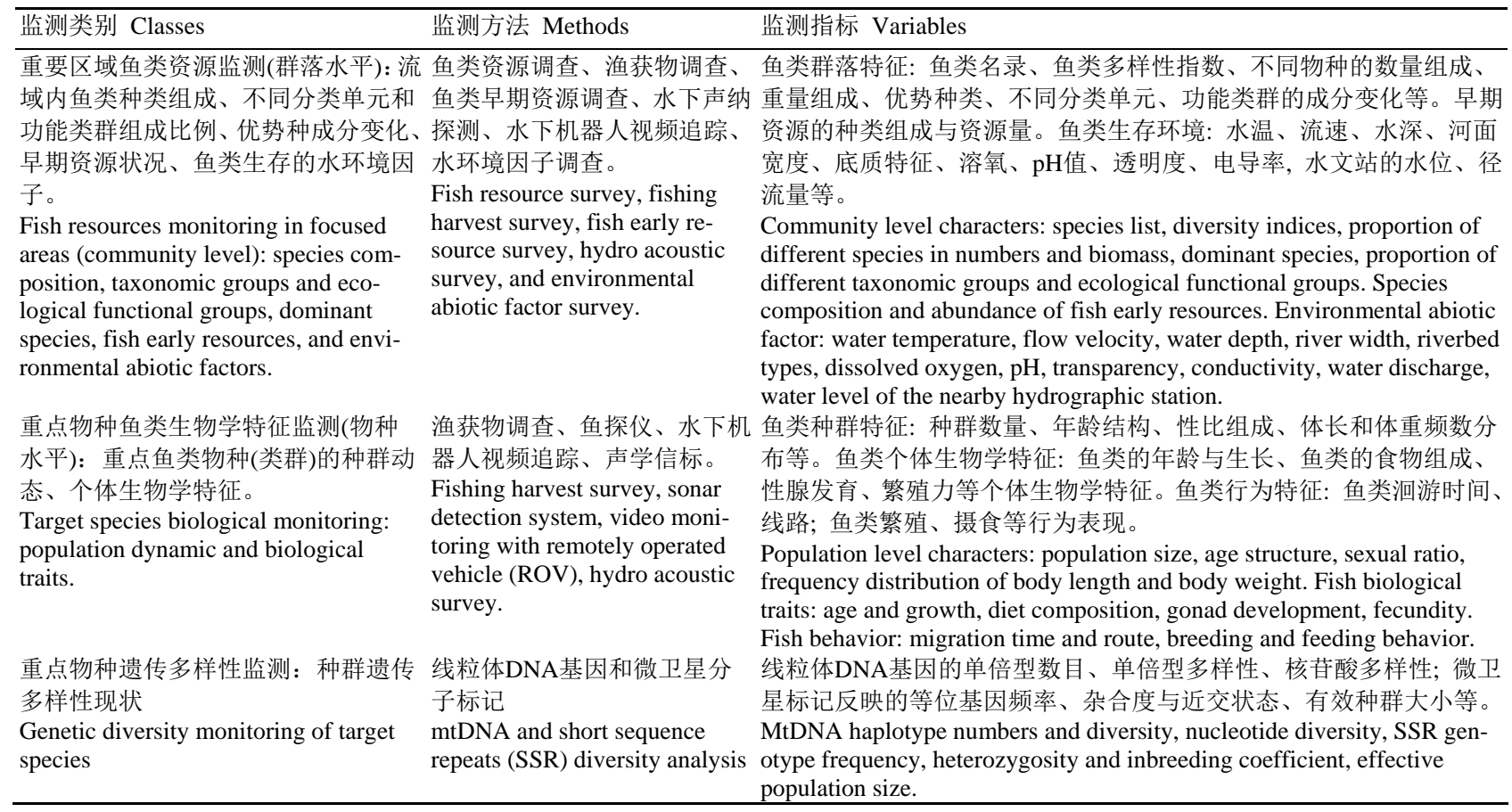


微卫星标记等分析其遗传多样性现状。

第三, 实现资源共享, 推动公众参与, 服务国 家建设。为更好地发挥专项网的公益性, 内陆水体 鱼类专项网将整理各流域监测数据及影像资料等 并通过网络共享, 带动公众参与到环境保护、鱼类 多样性保护、资料收集的各项进程。同时深入研究 鱼类多样性的维持机制, 提高我国生物多样性科学 研究水平, 为我国鱼类多样性保护、涉水工程开发 的决策提供依据。

目前有关我国内陆水体鱼类多样性的监测工 作在不同的水系、不同的区域均有一定程度的开展, 如珠江水系对鱼类早期资源的监测，长江流域“三峡 工程生态与环境监测系统”中水生动物和渔业资源 的监测, 农业部“渔业资源与环境监测”等。已有的这 些监测工作服务于不同的目的，积累了大量的资料。 但是, 目前还缺乏全国性的综合监测网络。中国内陆 水体鱼类多样性监测网是在中国生物多样性监测与 研究网络(Sino BON)的框架内搭建内陆水体鱼类多 样性监测的平台, 期望现有的区域性监测网络能够 相互合作, 通过数据共享, 形成全国性的监测网络, 为我国生物多样性的研究与保护做出贡献。

\section{参考文献}

Barko VA, Herzog DP, Hrabik RA, Scheibe JS (2004) Relationship among fish assemblages and main-channel-border physical habitats in the unimpounded upper Mississippi River. Transactions of the American Fisheries Society, 133, 371-384.

Chen YY (1990) Some aspects on biological diversity of freshwater ecosystem. Bioscience Communciation, 2, 197200. (in Chinese) [陈宜瑜 (1990) 淡水生态系统中的若干 生物多样性问题. 生物科学信息, 2, 197-200.]

Fewster RM, Buckland ST, Siriwardena GM, Baillie SR, Wilson JD (2000) Analysis of population trends for farmland birds using generalized additive models. Ecology, 81, 1970-1984.

Froese R, Pauly D (2016) FishBase. World Wide Web electronic publication. www.fishbase.org, version (10/2016).

Giles N, Sands R, Fasham M (2005) Fish. In: Handbook of Biodiversity Methods: Survey, Evaluation and Monitoring (eds Hill D, Fasham M, Tucker G, Shewry M, Shaw P), pp. 368-386. Cambridge University Press, Cambridge..

Hare SR, Mantua NJ (2000) Empirical evidence for North Pacific regime shifts in 1977 and 1989. Progress in Oceanography, 47, 103-145.

HJ 710.7-2014 (2014) Technical Guidelines for Biodiversity Monitoring-Inland Water Fish. China Environmental Science Press, Beijing. (in Chinese) [HJ 710.7-2014 (2014) 生
物多样性观测技术导则: 内陆水域鱼类. 中国环境科学 出版社, 北京.]

Karr JR (1981) Assessment of biotic integrity using fish communities. Fisheries, 6(6), 21-27.

Karr JR (1991) Biological integrity: a long-neglected aspect of water resource management. Ecological Applications, 1, 66-84.

Killgore KJ, Hoover JJ, George SG, Lewis BR, Murphy CE, Lancaster WE (2007) Distribution, relative abundance and movements of pallid sturgeon in the free-flowing Mississippi River. Journal of Applied Ichthyology, 23, 476-483.

Ma KP (2015) Biodiversity monitoring in China: from CForBio to Sino BON. Biodiversity Science, 23, 1-2. (in Chinese) [马克平 (2015) 中国生物多样性监测网络建设：从 CForBio到Sino BON. 生物多样性, 23, 1-2.]

Miller DL, Hughes RM, Karr JR, Leonard PM, Moyle PB, Schrader LH, Thompson BA, Daniels RA, Fausch KD, Fitzhugh GA, Gammon JR, Halliwell DB, Angermeier PL, Orth DJ (1988) Regional applications of an index of biotic integrity for use in water resource management. Fisheries, 13(5), 12-20.

Miranda LE, Killgore KJ (2014) Fish depth distributions in the Lower Mississippi River. River Research and Applications, 30, 347-359.

Nakano SI, Yahara T, Nakashizuka T (2012) The Biodiversity Observation Network in the Asia-Pacific Region: Toward Further Development of Monitoring, Ecological Research Monographs, Springer, Tokyo.

Pereira HM, Ferrier S, Walters M, Geller GN, Jongman RHG, Scholes RJ, Bruford MW, Brummitt N, Butchart SHM, Cardoso AC, Coops NC, Dulloo E, Fith DP, Freyhof J, Gregory RD, Heip C, Höft R, Hurtt G, Jetz W, Karp DS, McGeoch MA, Obura D, Onoda Y, Pettorelli N, Reyers B, Sayre R, Scharlemann JPW, Stuart SN, Turak E, Walpole M, Wegmann M (2013) Essential biodiversity variables. Science, 339, 277-278.

Sarkar UK, Pathak AK, Sinha RK, Sivakumar K, Pandian AK, Pandey A, Dubey VK, Lakra WS (2012) Freshwater fish biodiversity in the River Ganga (India): changing pattern, threats and conservation perspectives. Reviews in Fish Biology and Fisheries, 22, 251-272.

Scholes RJ, Mace GM, Turner W, Geller GN, Jürgens N, Larigauderie A, Muchoney D, Walther BA, Mooney HA (2008) Toward a Global Biodiversity Observing System. Science, 321, 1044-1045.

Scholes RJ, Walters M, Turak E, Saarenmaa H, Heip CH, Tuama ÉÓ, Faith DP, Mooney HA, Ferrier S, Jongman RH, Harrison IJ, Yahara T, Pereira HM, Larigauderie A, Geller G (2012) Building a global observing system for biodiversity. Current Opinion in Environmental Sustainability, 4, 139-146.

Steuck MJ, Yess S, Pitlo J, Van Vooren A, Rasmussen J (2010) Distribution and Relative Abundance of Upper Mississippi River Fishes. Upper Mississippi River Conservation Committee, Onalaska, Wisconsin.

(责任编委: 陈小勇 责任编辑: 时意专) 